\title{
Summation of undetected excitation following extinction of the CER
}

\author{
JEAN S. HENDRY \\ Princeton University, Princeton, New Jersey
}

\begin{abstract}
The effects of compounding two conditioned stimuli (CSs), each of which had been extinguished to varying degrees in different groups of rats given identical acquisition training, was examined within the conditioned emotional response paradigm. Greater suppression to the compound than to the individual CSs was observed following 6, 12, 48, 72, and 96 stimulus extinction trials, although after only 12 extinction trials suppression to the individual stimuli was no longer observed. The amount of compound suppression decreased progressively as the number of extinction trials increased until, after 120 extinction trials on each stimulus, the compound no longer elicited observable suppression. Control group data indicated that the observed summation effect could not be attributed to disinhibition. The possible role of the summation of undetected excitation in studies examining configural conditioning, avoidance conditioning and reinstatement is discussed briefly.
\end{abstract}

Summation effects in which the compounding of two excitatory stimuli produces greater suppression than either stimulus alone are well documented in the CER literature (Henderson, 1975; Miller, 1969; Rescorla, 1970; Van Houten, O'Leary \& Weiss, 1970; Wagner, 1971). However, relatively little work has focused on the effect of compounding two stimuli that have been independently conditioned and extinguished to the degree that they no longer elicit measurable suppression. Summation effects with respect to extinguished stimuli have typically been examined either during the extinction process or following a minimum number of extinction trials when the individual stimuli were still moderately excitatory (Reberg \& Black, 1969; Weiss \& Emurian, 1970). Reberg (1972), however, examined summation effects using a compound composed of one stimulus that had been extinguished to a level at which it no longer elicited measurable suppression and another stimulus that was moderately excitatory. It was reported that the compound elicited substantial suppression and that the degree of suppression was significantly greater than that elicited by either of the individual stimuli. Reberg suggested that the apparently extinguished stimulus had retained undetected

This paper was presented at the 52nd Annual Meeting of the Eastern Psychological Association, New York, April, 1981. The studies formed part of a dissertation submitted to Princeton University in partial fulfillment of the requirements for the $\mathrm{PhD}$ degree. The author is grateful to her advisor, L. J. Kamin, for his guidance and assistance throughout the course of the research. Requests for reprints should be sent to Jean S. Hendry, Department of Pharmacology, Medical College of Virginia, Box 613 MCV Station, Virginia Commonwealth University, Richmond, Virginia 23298. excitation which summed with that of the moderately excitatory stimulus to produce the marked suppression.

The present experiment was designed to examine the effect of compounding two stimuli that have been independently conditioned to a similar degree and extinguished to varying degrees beyond the point at which they no longer elicited measurable excitation.

\section{METHOD}

\section{Subjects}

The subjects were 48 experimentally naive male albino rats obtained from Holtzman Co., Madison, Wisconsin. They were reduced to $75 \%$ of ad-lib weight and maintained on a 24 -h feeding schedule throughout the study.

\begin{abstract}
Apparatus
The apparatus consisted of eight standard operant conditioning units individually housed in sound- and light-resistant chambers. The ceiling and two side walls of the operant units were made of clear acrylic plastic, with an additional sheet of frosted translucent plastic covering the ceiling. The two end walls were aluminum, with one wall containing a food cup, a loudspeaker, and a lever. The speaker permitted the presentation of an $80-\mathrm{dB}$ white noise. The floor of the units consisted of stainless steel grid bars connected to a Grason-Stadler shock generator (E1064GS), through which a $.5-\mathrm{sec}$ shock with an intensity of $1.0 \mathrm{~mA}$ could be presented. A 7.5-W bulb was mounted above the ceiling of each unit. The experiment was conducted with the units in complete darkness. Experimental events were controlled and recorded automatically by solid state and relay equipment located in an adjoining room.
\end{abstract}

\section{Procedure}

Following a single session of magazine and barpress training, all subjects were given 3 consecutive days of a 2-h session of barpress training under a 2.5 -min variable-interval food-reinforcement schedule. The reinforcer was a $45-\mathrm{mg}$ food pellet. The daily 
2-h barpressing sessions continued throughout the remainder of the experiment.

For the 2 days following preliminary training, all subjects were given, in each daily session, 3-min presentations of the light CS and the noise CS, superimposed on the ongoing operant behavior. The purpose of the pretest sessions was to assess the unconditioned responding to these two stimuli. Two light and two noise presentations were given in an alternating order in each session. The stimuli were presented in the same order on each of the pretest sessions.

Following pretesting, the subjects were randomly assigned to four experimental groups and to two control groups, with the restriction that half of the subjects in each group had received light as the first pretest trial and the other half had received noise as the first pretest trial. There were eight rats in each of the six groups. The four experimental groups received two shock-reinforced noise presentations and two shock-reinforced light presentations daily for 4 days. The two stimuli were presented for $3 \mathrm{~min}$ each in an alternating order, and each presentation was immediately followed by the $.5-\mathrm{sec}$ US. During the 4 days of acquisition, Control Group L6/48 received two shock-reinforced light presentations daily and Control Group N72/120 received two shockreinforced noise trials daily.

Following acquisition training, the groups were extinguished to the same stimuli to which they had been trained. The four experimental groups were each extinguished to light and to noise, but differed with respect to the number of extinction trials given to each stimulus. Groups 6,12 , and 48 were given 6,12 , and 48 extinction trials, respectively, to the two stimuli. Group $72 / 120$ received a total of 120 extinction trials to each stimulus. Control Group L6/48 was given a total of 48 extinction trials to light and Control Group N72/120 was given a total of 120 extinction trials to noise. In the extinction phase of the study, each of the experimental groups was given 6 extinction trials of light and 6 of noise in alternating order during each daily session. The control groups, which were undergoing extinction on a single element, were given a total of 6 extinction trials on their respective stimuli in each daily session.

Testing consisted of a single presentation of the nonreinforced light-noise compound, followed by nonreinforced presentations of the separate stimuli. Groups 6,12 , and 48 were tested on the compound and on the individual stimuli in the same daily session following the presentation of the last extinction trials in each group. Group $72 / 120$ was tested on three occasions during the course of extinction. This group was initially tested on the compound alone after 72 and after 96 extinction trials and on the compound and the individual stimuli after 120 extinction trials on each stimulus. This made possible the monitoring, within a single group, of suppression to the compound after extended extinction to the separate stimuli.

The two control groups were also tested three times during the course of extinction. Oroup L6/48 was tested on the compound and the individual stimuli after 6,12 , and 48 extinction trials of light. Group N72/120 was tested on the compound after 72 and 96 extinction trials of noise and on the compound and the individual stimuli after 120 noise extinction trials.

On the day following the last test series in each group, four nonreinforced compound trials were presented in the course of a 2-h experimental session. The Annau and Kamin (1961) suppression ratio was used to assess conditioning throughout the study. The ratio is of the form $B /(A+B)$, where $B$ represents the rate of barpressing during the $C S$ and $A$ represents the rate of barpressing in the 3-min interval immediately preceding the CS.

\section{RESULTS}

\section{Pretest}

There was slight, but significant, suppression to the CSs during pretesting. Pooling all groups, the mean suppression ratio was .40 on the first light presentation and .48 on the first noise presentation. By the final pretest trials, suppression was .44 to light and .54 to noise. There was significantly more unconditioned suppression to light than to noise $[F(1,42)=25.37, \mathrm{p}<.001]$, obviously reflecting a difference in the salience of the two stimuli.

\section{CER Acquisition}

During this phase, Groups $6,12,48$, and $72 / 120$ were treated identically; each of these groups received two reinforced light CSs and two reinforced noise CSs each day, for 4 days. The acquisition of suppression to each stimulus for the four groups, pooled, is plotted in the upper panel of Figure 1. The acquisition curves for light and for noise were virtually identical in the four experimental groups. As the pooled acquisition data reveal, the curves are quite typical, with suppression rapidly acquired to each stimulus and then attenuated slightly. A within-subject analysis of variance revealed that the effects of stimulus $[F(1,7)=6.23, p<.05]$ and of trials $[F(7,49)=71.92, p<.001]$ were significant. Both stimuli acquired significant levels of suppression during acquisition, although the degree of suppression was significantly greater for light than for noise throughout. A mixed analysis of variance conducted on the final acquisition trials of light and of noise in each of the four experimental groups indicated no significant differences, however.

The acquisition curves for the control groups trained to only one CS (lower panels of Figure 1) were similar to those for the groups trained to both CSs. A mixed analysis of variance comparing the light acquisition data for Group L6/48 (left panel)
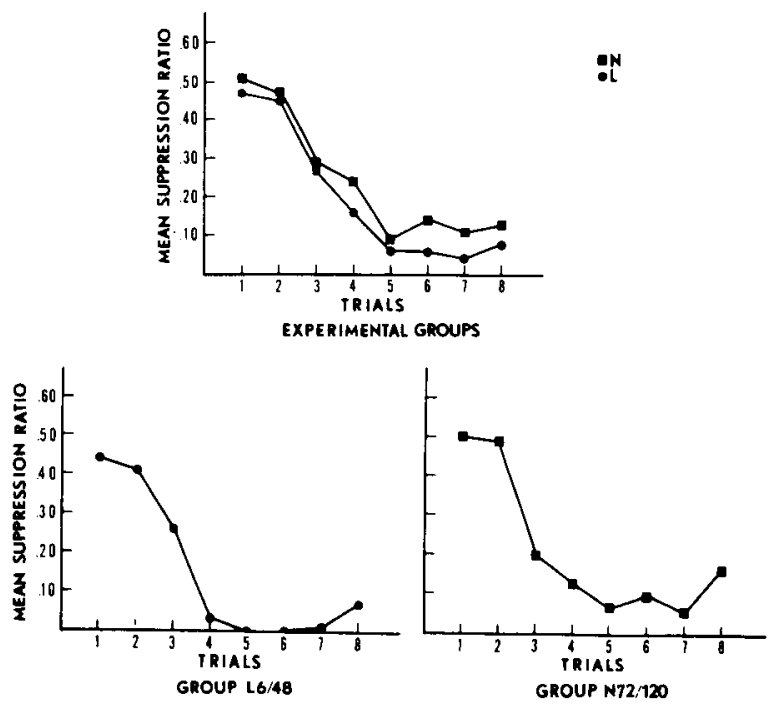

Figure 1. Mean suppresslon ratlos for the four experimental groups, pooled (upper panel), and for each of the control groups (lower panels) during acquisition training. $\mathbf{N}$ (squares) represents noise trials, and L (circles) represents light trials. 
with the pooled experimental group light acquisition data revealed only a significant effect of trials $[F(7,98)=55.71, \mathrm{p}<.001]$, indicating that suppression increased progressively during acquisition. A similar analysis conducted on the noise acquisition data for Group N72/120 (right panel) and the pooled experimental groups also revealed a significant effect of trials only $[F(7,98)=53.40, p<.001]$. Additional analyses of variance conducted on the last acquisition trials indicated that suppression on the final light trial in Group L6/48 and on the final noise trial in Group N72/120 did not differ significantly from the terminal suppression values observed to each stimulus in the experimental groups trained to both stimuli.

\section{Extinction}

The extinction curves for each stimulus are plotted in the upper panel of Figure 2, pooled for the four groups that had been trained to both stimuli. Since the groups differed with respect to the number of extinction trials, this means that for Extinction Trials 1 through 6, the $N$ is 32 ; for Extinction Trials 7 through 12, the $N$ is 24 animals; for Extinction Trials 13 through 48 , the $\mathbf{N}$ is 16 ; and for Trials 49 through 120 , the data are from the eight animals in Group 72/120. Note that the data in this figure are plotted in blocks of 2 trials for the first 30 trials of extinction to each stimulus and in blocks of 15 trials for the remainder of extinction, with the exception of Oroup L6/48, for which the last block of trials consists of 18 trials.

There were no obvious differences among the four groups in the rate of extinction to either stimulus.

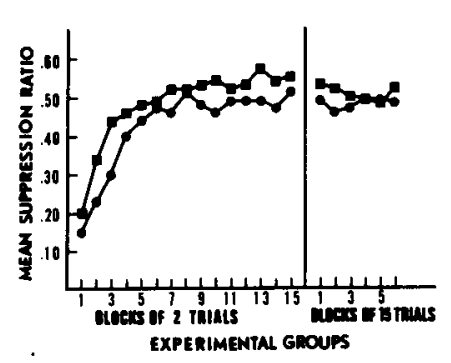

:

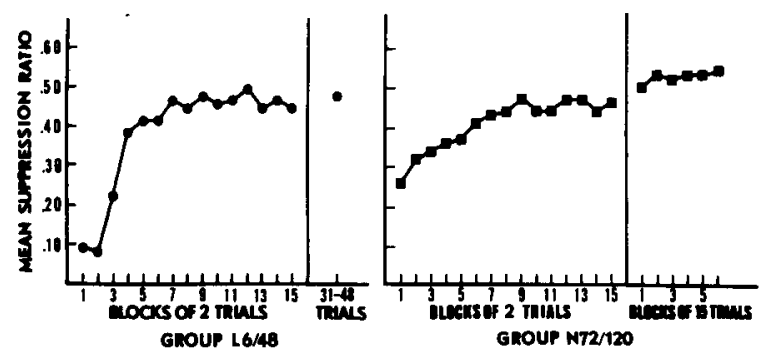

Figure 2. Mean suppreaclon ratios for the four experimental group, pooled (upper panel), and for each of the control groups (lower pasela) durias exthetion. N (aguarea) represents nolve thale, and I (elreled) represents tight trith.
As the pooled data show, extinction to each stimulus was quite rapid, particularly so in the case of noise. An analysis of variance on the pooled data conducted at several points during extinction revealed that, in fact, noise extinguished at a significantly faster rate than light $[F(1,7)=7.51, p<.05]$. In addition, the analysis revealed a significant effect of trials $[F(4,28)=102.99, p<.001]$, indicating that suppression attenuated to both stimuli as extinction continued. Inspection of the curves suggests that extinction to each stimulus was nearing completion after some 12 to 16 extinction trials with suppression ratios approximating .50 .

The extinction curves for light in Group L6/48 and for noise in Group N72/120 are presented separately in the lower left and right panels, respectively, of Figure 2. The extinction curves for these two groups were similar to those for the corresponding CSs in the experimental groups, although a mixed analysis of variance conducted at several points during extinction revealed that, in Group L6/48, light extinguished at a significantly slower rate than it did in the pooled experimental groups $[F(1,14)=5.39$, $\mathrm{p}<.05]$. The analysis also revealed a significant effect of trials $[F(7,98)=77.97, p<.001]$, indicating that suppression attenuated progressively as extinction continued. A similar analysis conducted on the noise extinction data for Group N72/120 and the pooled experimental groups revealed a significant effect of trials only $[F(9,126)=14.01, p<.001]$.

The mean suppression ratios for each group on the last extinction trials for each stimulus before testing are presented in Figure 3. These data clearly reflect the trends previously described. For the four experimental groups (upper panel), extinction to light and to noise was rapid, with ratios approaching .50 after 12 extinction trials. The mean suppression ratios following 72 extinction trials in Group 72/120 actually indicate slightly more suppression to light than was observed in Group 12 after 12 extinction trials, and slightly less suppression to noise. However, there is clearly no further weakening of suppression detectable after 12 extinction trials in the between-groups comparison.

The data on the last extinction trials of light and of noise prior to testing for each of the four groups (including only the data at 72 trials in Group 72/120) were submitted to a mixed analysis of variance. The only significant effect was the main effect of group $[F(3,28)=4.37, p<.025]$. Since each group differed with respect to the number of element extinction trials, this significant effect indicates that the stimuli extinguished over trials. In addition, the extinction data within Group 72/120 were submitted to a within-subject analysis of variance. There were no significant effects. That is, no further extinction could be demonstrated in a within-subject compari- 
son between 72 and 120 extinction trials. The 120-trial data for Group 72/120 and the data for Group 12 were also submitted to a separate mixed analysis of variance. Again, no significant effects were found, indicating that no additional stimulus extinction occurred between 12 and 120 trials.

The extinction data for the two control groups (lower panel) are very similar to the data for the experimental groups. Within Group L6/48, extinction to light can be observed, and the ratios after 6,12 , and 48 trials are very close to those observed in Experimental Groups 6, 12, and 48. Three separate $t$ tests indicated no significant differences. Within Group N72/120, extinction to noise appears complete by the end of 72 trials with no further weakening of suppression between 72 and 120 trials. When noise extinction data for Groups N72/120 and 72/
120 were submitted to a mixed analysis of variance, Group $72 / 120$ was found to be, overall, significantly more suppressed $[F(1,14)=8.03, p<.025]$. Though statistically significant, the magnitude of the effect is not large, and by 120 extinction trials both groups had identical suppression ratios to noise. This effect may reflect a genuine tendency for a group conditioned to two stimuli (and thus experiencing twice as many shocks) to extinguish a little less rapidly than a group conditioned to one stimulus. However, sampling error is also possible, particularly since no analogous effect was detected in Group L6/48.

\section{Test Data}

The mean suppression ratios for all groups on the tests of the compound and the individual stimuli are presented in Figure 3. It will be recalled that, after
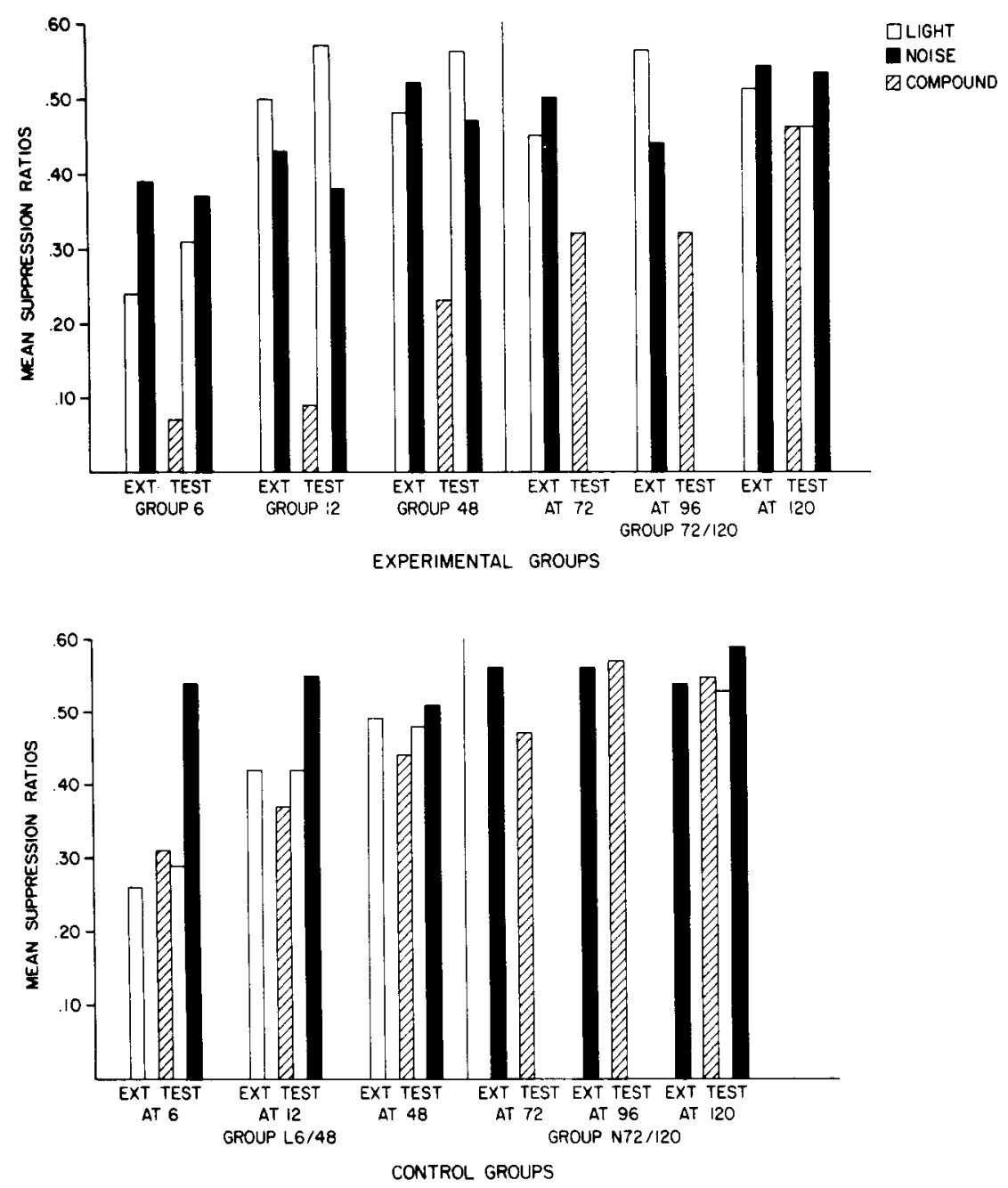

Figure 3. Mean suppression ratios for the last stimulus extinction trials (EXT) prior to compound testing and on the tests of the compound, light and noise, for each of the experimental groups (upper panel) and the control groups (lower panel). The open bars represent the light, closed bars represent the noise, and slashed bars represent the compound. Groups $72 / 120, \mathbf{L 6} / 48$, and $\mathbf{N 2} / 120$ were each tested at three intervals during extinction. 
72 and 96 extinction trials in Groups $72 / 120$ and $\mathrm{N} 72 / 120$, only the compound was tested. The test data for the four experimental groups (upper panel) revealed that suppression to the elements was scarcely detectable after 12 extinction trials and not at all observable after 48 trials. However, compound test suppression was observed after 72 and even 96 extinction trials, long after suppression to the individual stimuli seemed to be completely extinguished. A mixed analysis of variance of the test data for the compound, light and noise, for Groups 6, 12, and 48 revealed a significant effect of stimulus $[F(2,42)=$ $30.89, p<.001]$ and of group $[F(2,21)=6.77, p<.01]$. A subsidiary Newman-Keuls multiple range test revealed that suppression was significantly greater on the tests of the compound than on the tests of the individual stimuli ( $\alpha=.05$ ). The significant group effect merely indicated that overall test suppression decreased as the number of extinction trials increased. A within-subject analysis of variance for Group $72 / 120$ on the data obtained on the compound test and on the stimulus extinction trials immediately preceding the compound test revealed a significant effect of stimulus $[F(2,14)=4.41, p<.05]$. A Newman-Keuls multiple range test indicated that compound suppression was significantly greater than suppression to the individual stimuli $(\alpha=.05)$.

The magnitude of the summation effect, evidenced by compound test suppression, was larger after 6 , 12 , and 48 extinction trials on the individual stimuli and smaller after 72 and 96 extinction trials. Cl’arly, suppression to the compound progressively dec: ased as the number of stimulus extinction trials increased. An analysis of variance on the compound test data for Groups 6, 12, 48, and 72/120 (including only the compound test data after 72 extinction trials)

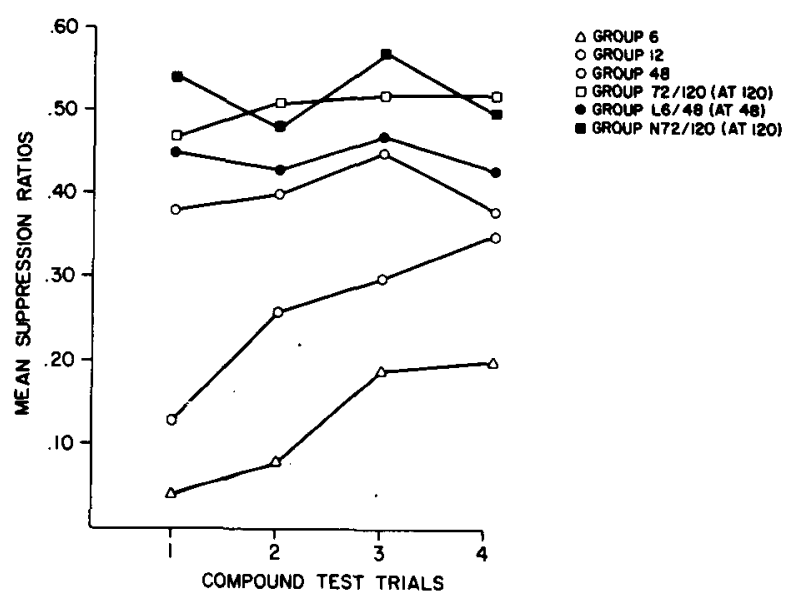

Figure 4. Mean suppression ratios for Group 6 (open triangles), Group 12 (open hexagon), Group 48 (open circles), Group 72/120 (open squares), Group L6/48 (closed circles), and Group N72/120 (closed squares) on the four tests of the compound obtained on the day followise the presentation of the last stimulus extinction trials. revealed that the loss of compound suppression was significant $[F(3,28)=5.24, p<.01]$. A within-subject analysis of variance on the three compound tests in Group 72/120 revealed that the loss of suppression across the three tests was not significant, despite the fact that the compound was virtually neutral after 120 extinction trials, with a ratio of .46 . Obviously, the loss of suppression to the compound was not significant within this group because compound suppression was already well attenuated after 72 extinction trials.

\section{Control for Disinhibition}

The compound tests of the two control groups (Figure 3, lower panel) provide data relevant to the possibility that suppression to the compound in the experimental groups may be produced by a disinhibition effect. It is conceivable that the presentation of a compound stimulus to animals previously exposed to only the isolated stimulus elements may constitute the presentation of a novel, extraneous stimulus that could disinhibit the extinguished response to the individual stimuli.

Tests of noise as a possible disinhibitory stimulus were examined in Group L6/48. This group was tested on the compound after 6,12 , and 48 extinction trials to light. Data on each of these three tests were compared with the compound test data for the appropriate experimental groups. Suppression to the compound on each of the three comparisons was found to be significantly greater for the experimental group than for the control group $[\mathrm{t}(14)=4.25$, $\mathrm{p}<.001$, after 6 extinction trials; $\mathrm{t}(14)=6.82$, $\mathrm{p}<.001$, after 12 extinction trials; and $\mathrm{t}(14)=3.20$, $\mathrm{p}<.01$, after 48 extinction trials]. Thus, at no point during extinction in Group L6/48 did the simultaneous presentation of the extraneous noise with the light CS produce as much suppression as was observed in the appropriate experimental group.

Tests of light as the possible disinhibitory stimulus were examined in Group N72/120. This group was tested on the compound after 72, 96, and 120 extinction trials to noise. Data for each of these three tests were compared with the compound test data for Group 72/120. A mixed analysis of variance indicated that the effect of group was significant $[F(1,14)=$ $11.90, \mathrm{p}<.01$ ], with greater compound suppression in Group $72 / 120$ than in Group N72/120. Thus, once again there is no evidence for a disinhibition effect.

An alternative way to assess the possibility that disinhibition was responsible for the suppression observed on the compound tests in the experimental groups is to determine if any disinhibition occurred within each of the control groups as a function of adding the novel, extraneous stimulus. To assess disinhibition within the control groups, a comparison was made between suppression on the compound test 
and on the immediately preceding element extinction trial. If any disinhibition occurred, suppression to the compound should be greater than suppression to the element. As the data in Figure 3 show, there was no consistent tendency within either control group for suppression ratios to differ between the last stimulus extinction trial and the compound tests. A within-subject analysis of variance indicated no significant effect of stimulus in either group. That is, the addition of an extraneous stimulus did not, in any instance, significantly alter the responding that had been observed one trial earlier to a partially (or almost totally) extinguished CS.

\section{Final Compound Tests}

The last experimental session for all groups consisted of four nonreinforced compound presentations. The mean suppression ratios for these four trials for all groups are presented in Figure 4. The data for the first trial are very similar to the compound data depicted in Figure 3. However, with repeated testing, suppression to the compound tended to diminish in the experimental groups. When the data for the four experimental groups were submitted to a mixed analysis of variance, the effects of group $[F(3,28)=15.22, p<.001]$ and of trials $[F(3,84)=9.92, p<.001]$ were found to be significant. This merely confirms that, among the experimental groups, suppression to the compound was greatest in the groups with fewer element extinction trials and that suppression diminished with repeated nonreinforced testing. The control group data in Figure 4 reveal that suppression to the compound was not significant on any of the four trials.

\section{DISCUSSION}

The basic results of this experiment are straightforward. Following eight acquisition trials to a CS, nonreinforced presentations of that CS produced rapid and orderly extinction. Extinction appeared to be virtually complete after only 12 trials when assessed by observing the response to the nonreinforced CS. Evidence of further element extinction could be detected neither between groups nor within groups. However, significant suppression was observed when two "extinguished" CSs were compounded after $12,48,72$, and 96 extinction trials. After 6 extinction trials, the excitatory strengths of the two partially extinguished CSs combined to produce a clear summation effect. It is also reasonable to conclude that, after $12,48,72$, and 96 trials, summation of excitation was still occurring, although the excitatory properties of the individual stimuli were not detected when the stimuli were presented singly. Thus, the compounding of apparently extin- guished CSs provides a sensitive test of their "latent" excitatory properties.

The fact that suppression to the compound progressively weakened as element extinction was continued "beyond zero," or the point at which suppression could no longer be detected, indicates that the individual stimuli had continued to lose excitatory value as a consequence of repeated nonreinforced presentations. By 120 extinction trials of each stimulus, virtually all excitatory strength had disappeared and the test of the compound at this point revealed no suppression. Thus, we might assume that by the 120 th element extinction trials, there was no "undetected excitation" left to sum.

The suppression observed on the compound tests in the experimental groups clearly may not be attributed to a simple disinhibition effect. Each test of the compound in these groups revealed consistently greater suppression than that found in the appropriate control group. In addition, within the control groups, compound suppression did not differ significantly from suppression on their respective preceding element extinction trials.

It should be noted that repeatedly testing the compound in the disinhibition control groups could have reduced the novelty of the disinhibitory stimuli and, consequently, their disinhibitory effects (Pavlov, 1927). Brimer (1970), however, reported that familiarity with the disinhibitor does not reduce the magnitude of disinhibition. In any event, there is no evidence, even in the first "pure" tests of the compound in the control groups, to suggest that the compound suppression in the experimental groups was at all influenced by disinhibition.

The robustness of the summation effect observed in the present study suggests that summation of this sort may be occurring unsuspectedly in many experimental contexts, possibly leading to errors in interpretation. Kamin and Idrobo (1978), for example, have suggested that configural conditioning may be an experimental situation in which summation effects occur. In configural conditioning, the independent elements constituting a compound stimulus merge into a configure with repeated reinforced compound presentations (Razran, 1965, 1971). As the configure develops, it acquires excitatory properties while the excitation that initially accrued to the separate elements disappears. In the Kamin and Idrobo study, which was similar in design to an earlier study reported as a demonstration of configural conditioning by Gray and Lethbridge (1976), the number of reinforced compound trials given to separate groups of rats was varied. Acquisition training within each group was followed by a test of the individual elements. The results of the study were similar to those reported by Gray and Lethbridge. Suppression to the compound was greater than sup- 
pression to the separate elements, and, in addition, suppression both to the compound and to the elements decreased as the number of reinforced compound trials increased. Kamin and Idrobo suggested that this pattern of responding might reflect a summation effect in which the excitation of the two elements sums when presented in compound to produce greater suppression than that produced by either element alone. In addition, these investigators noted that the loss of suppression to the elements (and, consequently, to the compound) with repeated reinforced compound trials may simply reflect the postasymptotic loss of suppression often observed in CER studies (Annau \& Kamin, 1961; Millenson \& Hendry, 1967). Thus, it appears that summation effects may be occurring in studies interpreted as demonstrations of configural conditioning.

There are also some paradoxical effects in the literature that could possibly reflect the summation of excitation of an "extinguished" CS with excitation of background cues. For example, Kamin, Brimer, and Black (1963) found that instrumental avoidance responding in a shuttlebox occurred despite the fact that fear of the CS signaling shock, as tested in a subsequent CER procedure, was extinguished. It is possible that the undetected excitation of the background cues in the shuttlebox may have summed with the undetected excitation of the "extinguished" CS when presented together to produce a level of fear of sufficient magnitude to evoke the avoidance response. It should be noted that the assessment of the fear-eliciting properties of the CS was made in a Skinner box, where the animals had never been shocked.

More recently, Bouton and Bolles (1979) examined the reinstatement of fear to an extinguished $\mathrm{CS}$ as a function of presenting the US after extinction training. A subsequent presentation of the CS indicated that fear was reinstated only when the reinstating shock was presented in the same context in which the CS was tested. Furthermore, if contextual stimuli were extinguished prior to testing, it was found that reinstatement was attenuated. These investigators. suggested that reinstatement might be the result of contextual cue excitation summing with the fear retained by the CS. It therefore appears likely that summation contributes, at least in part, to reinstatement effects.

In summary, the summation of undetected excitation which occurs when two "extinguished" CSs are compounded is a real and robust phenomenon. Ex- perimental designs that allow the possibility for this type of summation should include control groups appropriate for assessing the effect.

\section{REFERENCES}

Annau, Z., \& Kamin, L. J. The conditioned emotional response as a function of intensity of the US. Journal of Comparative and Physiological Psychology, 1961, 54, 428-432.

Bouton, M. E., \& Bolles, R. C. Role of conditioned contextual stimuli in the reinstatement of extinguished fear. Journal of Experimental Psychology: Animal Behavior Processes, 1979, 5, 368-378.

Brimer, C. J. Disinhibition of an operant response. Learning and Motivation, 1970, 1, 346-371.

Gray, T., Letheridae, D. A. Configural conditioning in the CER: Loss of element strength after repeated reinforced compound CS trials. Learning and Motivation, 1976, 7, 532-539.

Hendergen, R. W. Compounds of conditioned fear stimuli. Learning and Motivation, 1975, 6, 28-42.

Kamin, L. J., Brimer, C. J., \& Black, A. H. Conditioned suppression as a monitor of fear of the CS in the course of avoidance training. Journal of Comparative and Physiological Psychology, 1963, 56, 497-501.

Kamin, L. J., \& IDrobo, F. Configural conditioning in the CER: A possible artifact. Animal Learning \& Behavior, 1978, 6, 290. 293.

Millenson, J. R., \& Hendny, D. P Quantification of response suppression in conditioned anxiety training. Canadian Journal of Psychology, 1967, 21, 242-252.

Miller, L. Compounding of pre-aversive stimuli. Journal of the Experimental Analysis of Behavior, 1969, 12, 293-299.

Pavlov, I. P. [Conditioned reflexes] (G. V. Anrep, trans.). London: Oxford University Press, 1927.

RAzRAN, G. Empirical codification and specific theoretical implications of compound-stimulus conditioning: Perception. In W. F. Prokasy (Ed.), Classical conditioning. New York: AppletonCentury-Crofts, 1965.

Razran, G. Mind in Evolution. Boston: Houghton Mifflin, 1971.

Rebero, D. Compound tests for excitation in early acquisition and after prolonged extinction of conditioned suppression. Learning and Motivation, 1972, 3, 246-258.

Regero, D., \& Black, A. H. Compound testing of individually conditioned stimuli as an index of excitatory and inhibitory properties. Psychonomic Science, 1969, 17, 30-31.

Resconu, R. A. Reduction in the effectiveness of reinforcement after prior excitatory conditioning. Learning and Motivation, 1970, 1, 372-381.

Van Houten, R., O'Leary, K. D., \& We18s, S. J. Summation of conditioned suppression. Journal of the Experimental Analysis of Behavior, 1970, 13, 75-81.

WhGNer, A. R. Elementary associations. In H. H. Kendler \& J. T. Spence (Eds.), Essays in neobehaviorism: A memorial volume to Kenneth $W$. Spence. New York: Appleton-CenturyCrofts, 1971.

Weise, S. J., \& Eyurian, H. H. Stimulus control during the summation of conditioned suppression. Journal of Experimental Psychology, 1970, 85, $204-209$.

(Manuscript received August 31, 1981; revision accepted for publication May 24, 1982.) 